\title{
Injury of the Contralateral Lower Ascending Reticular Activating System by an Intracerebral Hemorrhage
}

A 56-year-old female patient underwent decompressive craniectomy for removal of an intracerebral hematoma in the right temporoparietal lobe and spontaneous subarachnoid hemorrhage due to a ruptured right middle cerebral artery bifurcation aneurysm at the department of neurosurgery of a university hospital (Fig 1A). At 3 weeks after onset, she was transferred to the rehabilitation department. The patient exhibited impaired consciousness, with a Glasgow Coma Scale score of 8 (eye opening, 3; best verbal response, 1 ; and best motor response, 4$)$. Seven agematched control subjects ( 3 men; mean age, 55.28 years) were recruited. All subjects provided signed, informed consent, and the study protocol was approved by our institutional review board.

Diffusion tensor imaging data were acquired at 3 weeks after onset by using a 6-channel head coil on a 1.5T Gyroscan Intera scanner (Philips Healthcare, Best, the Netherlands). For each of the 32 noncollinear diffusion-sensitizing gradients, we acquired 67 contiguous sections. Imaging parameters were as follows: $\mathrm{TR}=10,726 \mathrm{~ms}, \mathrm{TE}=76 \mathrm{~ms}, b=1000 \mathrm{~s} / \mathrm{mm}^{2}, \mathrm{NEX}=1$, section thickness $=2.3 \mathrm{~mm}$. Fiber tracking was performed by using a probabilistic tractography method based on a multifiber model (FSL, http://www.fmrib.ox.ac.uk/fsl; 5000 streamline samples; 0.5 -mm step lengths; curvature thresholds $=0.2$ ). The seed ROI was placed at the reticular formation of the pons, and the target ROI was placed at the intralaminar nuclei of the thalamus. ${ }^{1-3}$ Fractional anisotropy (FA) and tract volume (TV) of the lower portion of the ascending reticular activation system (ARAS) were measured. In the patient, TV of the left ARAS was decreased $>2$ SDs compared with that of control subjects. The left lower ARAS was thinner than the right one of the patient and those of control subjects.

In this study, by using diffusion tensor tractography, we evaluated the lower portion of the ARAS between the pontine reticular formation and the thalamus in a patient with brain injury.

http://dx.doi.org/10.3174/ajnr.A4446
Only the TV of the left lower ARAS was decreased compared with that in the control group. The TV is determined by the number of voxels contained within a neural tract; therefore, the decrement of the TV without a change of FA value appears to indicate injury of the left lower ARAS. On the configuration of diffusion tensor tractography, thinning of the left lower ARAS appears to coincide with the decrement of the TV of the left lower ARAS. It appears that the left lower ARAS might have been injured by the Kernohan notch phenomenon similar to an injury mechanism following transtentorial herniation by the right intracerebral hematoma. ${ }^{4}$ Our results suggest that analysis of the ARAS by using DTT would be helpful in elucidating the cause of impaired consciousness in patients with brain injury.

\section{ACKNOWLEDGMENTS}

This work was supported by the National Research Foundation of Korea Grant funded by the Korean Government (NRF2015R1A2A2A01004073).

\section{REFERENCES}

1. Jang SH, Kim HS. Aneurysmal subarachnoid hemorrhage causes injury of the ascending reticular activating system: relation to consciousness. AJNR Am J Neuroradiol 2015;36:667-71

2. Jang $\mathrm{SH}$, Kim SH, Lim HW, et al. Injury of the lower ascending reticular activating system in patients with hypoxic-ischemic brain injury: diffusion tensor imaging study. Neuroradiology 2014;56: 965-70

3. Jang SH, Kim SH, Lim HW, et al. Recovery of injured lower portion of the ascending reticular activating system in a patient with traumatic brain injury. Am J Phys Med Rehabil 2015;94:250-53

4. Jang SH, Lim HW, Yeo SS. Injury of the ascending reticular activating system by transtentorial herniation in a patient with intracerebral haemorrhage: a diffusion tensor tractography study. J Neurol Neurosurg Psychiatry 2015 Jan 30. [Epub ahead of print]

Department of Physical Medicine and Rehabilitation College of Medicine, Yeungnam University Daegu, Republic of Korea 


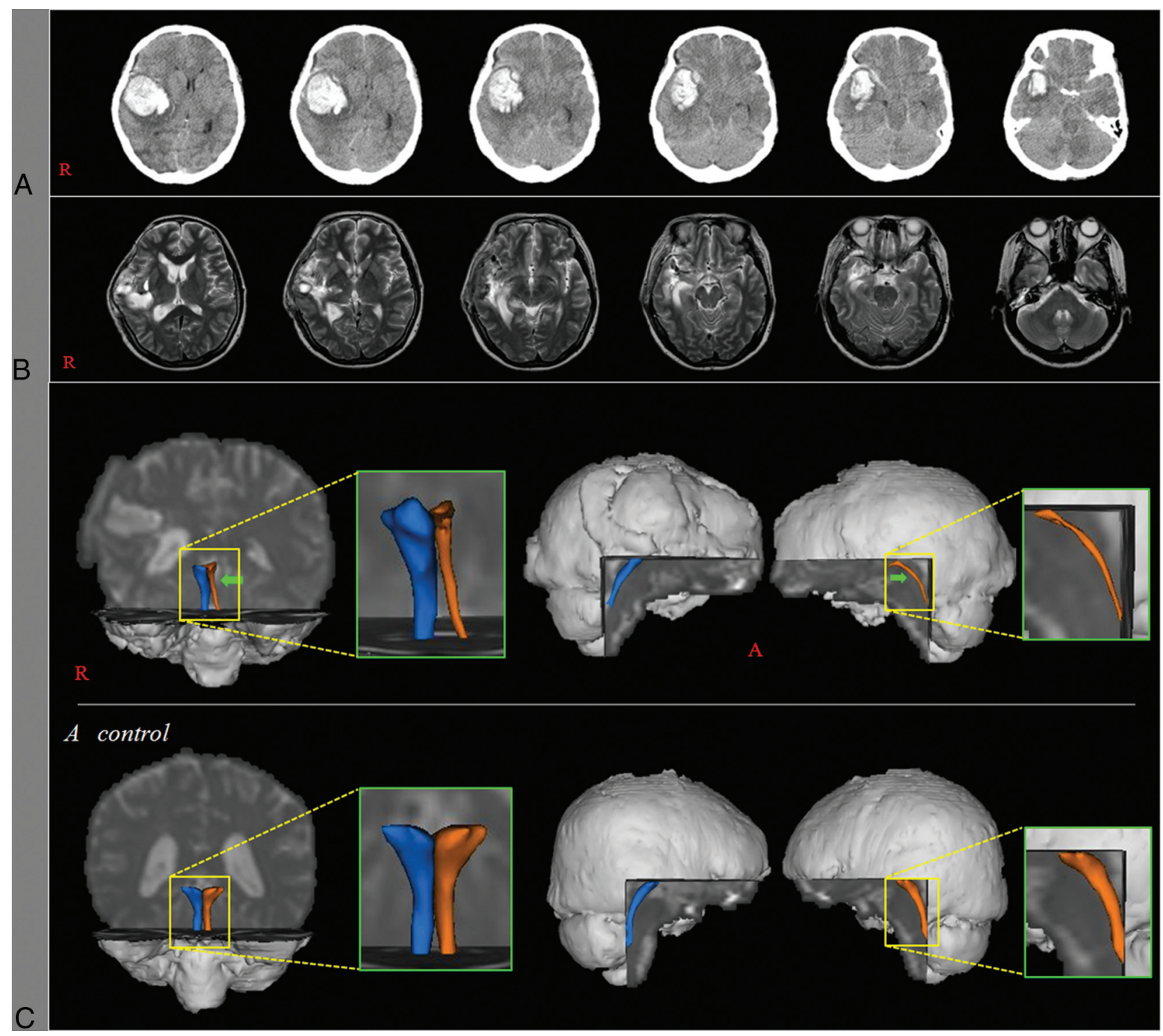

FIG 1. A, Brain CT images at onset show an intracerebral hemorrhage in the right frontotemporal lobes and subarachnoid hemorrhage. $B$, Brain MR images obtained at 3 weeks after onset show a malactic lesion in the right frontotemporal lobes. C, Results of diffusion tensor tractography. The left lower ascending reticular activating system is thinner than the right one of the patient and those of a control subject (a 54-year-old woman). 